EASD Procedia
EURODYN 2020

XI International Conference on Structural Dynamics M. Papadrakakis, M. Fragiadakis, C. Papadimitriou (eds.) Athens, Greece, 23-26 November 2020

\title{
UNCERTAINTIES IN WIND-INDUCED LOADS ON HYPERBOLIC PARABOLOID ROOFS: WIND-TUNNEL TESTS AND ANALYTICAL MODELS
}

\author{
Fabio Rizzo and Vincenzo Sepe
}

\author{
Department of Engineering and Geology, University "G. D'Annunzio" \\ Viale Pindaro 42, 65127 Pescara, Italy \\ \{fabio.rizzo, vincenzo.sepe\}@unich.it
}

Keywords: wind-tunnel tests, hyperbolic paraboloid roof, wind loads, pressure coefficients, experimental uncertainties.

\begin{abstract}
The design coefficients provided by a wind-tunnel campaign on in-scale models, e.g. the pressure coefficients discussed in this paper, are always affected by physiological and unavoidable uncertainties. On the other hand, wind-induced loads at the real scale (e.g. peak pressure coefficients) may be provided, with a prescribed confidence level, by analytical models that include stochastic characteristics of the wind field (mean values, standard deviation, skewness, kurtosis) obtained by wind-tunnel tests. Based on aerodynamic tests on hyperbolic paraboloid models in the boundary layer wind-tunnel of the CRIACIV (Italy), this paper discusses a typical example of experimental uncertainties, i.e. the asymmetries measured for geometries and wind directions nominally symmetric, and investigates their effects on peak pressure coefficients provided by analytical models. To this aim, experimental peak pressure coefficients and their analytical prediction are compared for symmetrical geometries under wind flow direction parallel to the symmetry axis, and it is noted that asymmetries shown by experimental tests may be reduced by appropriate analytical models, that can therefore contribute to filter out the effects of experimental uncertainties for design purposes.
\end{abstract}

ISSN:2311-9020 @ 2020 The Authors. Published by EASD Procedia.

Peer-review under responsibility of the Organizing Committee of EURODYN 2020. doi: $10.47964 / 1120.9290 .20259$ 


\section{INTRODUCTION}

Although computational fluid dynamics (CFD) has become more and more accurate and reliable in the last years, and can therefore be an invaluable tool in the preliminary design stage, wind tunnel tests on in-scale models are still the more reliable way to get the windinduced loads for structures with a complex shape.

Wind-tunnel tests, however, are always affected by several uncertainties, due to the measurements chain, to the scaling of the real building at the model size, to the geometrical details of the model and to its mechanical characteristics. The design coefficients provided by a wind-tunnel campaign, e.g. the pressure coefficients discussed in this paper, are therefore also affected by physiological and unavoidable uncertainties; this is the case, for example, of nominally symmetric and symmetrically loaded models for which wind-tunnel tests turn out instead asymmetric pressure fields.

As shown by the scientific literature, on the other hand, a sound prediction of windinduced loads at the real scale may benefit of analytical models (e.g. [1][2][3]) able to provide, for example, average or peak pressure coefficients with a prescribed confidence level. To this aim, analytical models may use the stochastic characteristics of the wind field (mean values, standard deviation, skewness, kurtosis) obtained by wind-tunnel tests. This point has been addressed in a recent paper of the writers [4].

This paper is based on experimental results on hyperbolic paraboloid models tested in the boundary layer wind-tunnel of the CRIACIV (Italy) [4-9] and is part of a wider research that also deals with non-structural aspects of the design $[10,11]$. The paper discusses a typical example of experimental uncertainties, i.e. the asymmetries measured for geometries and wind directions nominally symmetric, and investigates their effects on peak pressure coefficients provided by two of the analytical models discussed in [4], i.e. the classical Davenport model and the modified Hermite model discussed in [1].

\section{WIND-TUNNEL TESTS}

Aerodynamic tests on a large set of hyperbolic paraboloid roofs were described in previous papers of the writers [5-9]. Wooden in-scale models with 16 different geometries and 16 different wind angles were tested in the boundary layer wind tunnel of CRIACIV (Interuniversity Research Center on Building Aerodynamics and Wind Engineering), in Italy.

In particular, this paper is focused on results obtained by wind tunnel test on roofs with square and rectangular plan and eight different geometries are discussed here.

The models represent closed buildings, e.g. as those of sports halls, and this paper discusses the aerodynamic pressure field recorded on the roof surfaces. Due to the stiffness of the models, that can be considered as rigid, roof displacements and therefore aeroelastic effects are not considered; this is an usual step in the preliminary design of this kind of structures.

\subsection{The sample square and rectangular models}

Figure 1 describes the geometries discussed in Sect. 2.2 and Sect. 3.

The eight geometries considered here are denoted by three letters that refer to the model plan shape (square, $\mathrm{S}$, or rectangular, R), to the model height (higher, H, or lower, L), and to the roof curvature (with smaller curvature or flatter, F, or higher curvature, C), respectively.

As reported in fig. 2, the roof surface consists of 89 and 95 elementary regions for the square and rectangular plan, respectively. Pressure time-histories were recorded (details in [6]) for about 30 seconds with an acquisition frequency of about $250 \mathrm{~Hz}$ for several angles of inci- 
dence of wind. To explore situations nominally symmetric, only the wind directions $0^{\circ}$ and $90^{\circ}$ are discussed be ${ }^{1} \mathrm{w}$, as shown in fig. 1 .

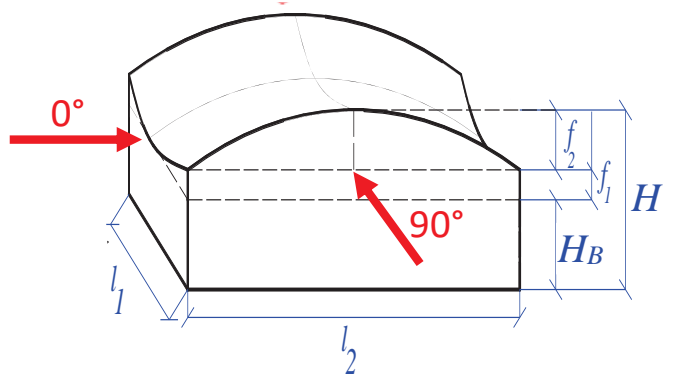

\begin{tabular}{ccccccc}
\hline model & $l_{1}[\mathrm{~cm}]$ & $l_{2}[\mathrm{~cm}]$ & $f_{1}[\mathrm{~cm}]$ & $f_{2}[\mathrm{~cm}]$ & $H[\mathrm{~cm}]$ & $H_{B}[\mathrm{~cm}]$ \\
\hline SLF & 80 & 80 & 2.67 & 5.33 & 21.33 & 13.33 \\
SHF & 80 & 80 & 2.67 & 5.33 & 34.66 & 26.66 \\
SLC & 80 & 80 & 4.44 & 8.89 & 26.66 & 13.33 \\
SHC & 80 & 80 & 4.44 & 8.89 & 39.99 & 26.66 \\
RLF & 40 & 80 & 2.67 & 5.33 & 21.33 & 13.33 \\
RHF & 40 & 80 & 2.67 & 5.33 & 34.66 & 26.66 \\
RLC & 40 & 80 & 4.44 & 8.89 & 26.66 & 13.33 \\
RHC & 40 & 80 & 4.44 & 8.89 & 39.99 & 26.66 \\
\hline
\end{tabular}

Figure 1: The eight sample models

\subsection{Pressure coefficients}

For the pressure tap of each elementary region of the roof, the measured pressure timehistory $p(t)$ is described by non-dimensional pressure coefficients $c_{p}(t)$, defined as

$$
c_{p}(t)=\frac{p(t)-p_{0}}{\frac{1}{2} \rho V_{m}^{2}}
$$

where $\rho$ denotes the air density and $V_{m}$ and $p_{0}$ the mean speed at the roof level and the static pressure of undisturbed flow, respectively. Positive and negative values of $c_{p}$ represent pressure larger than $p_{0}$ and suction, respectively.
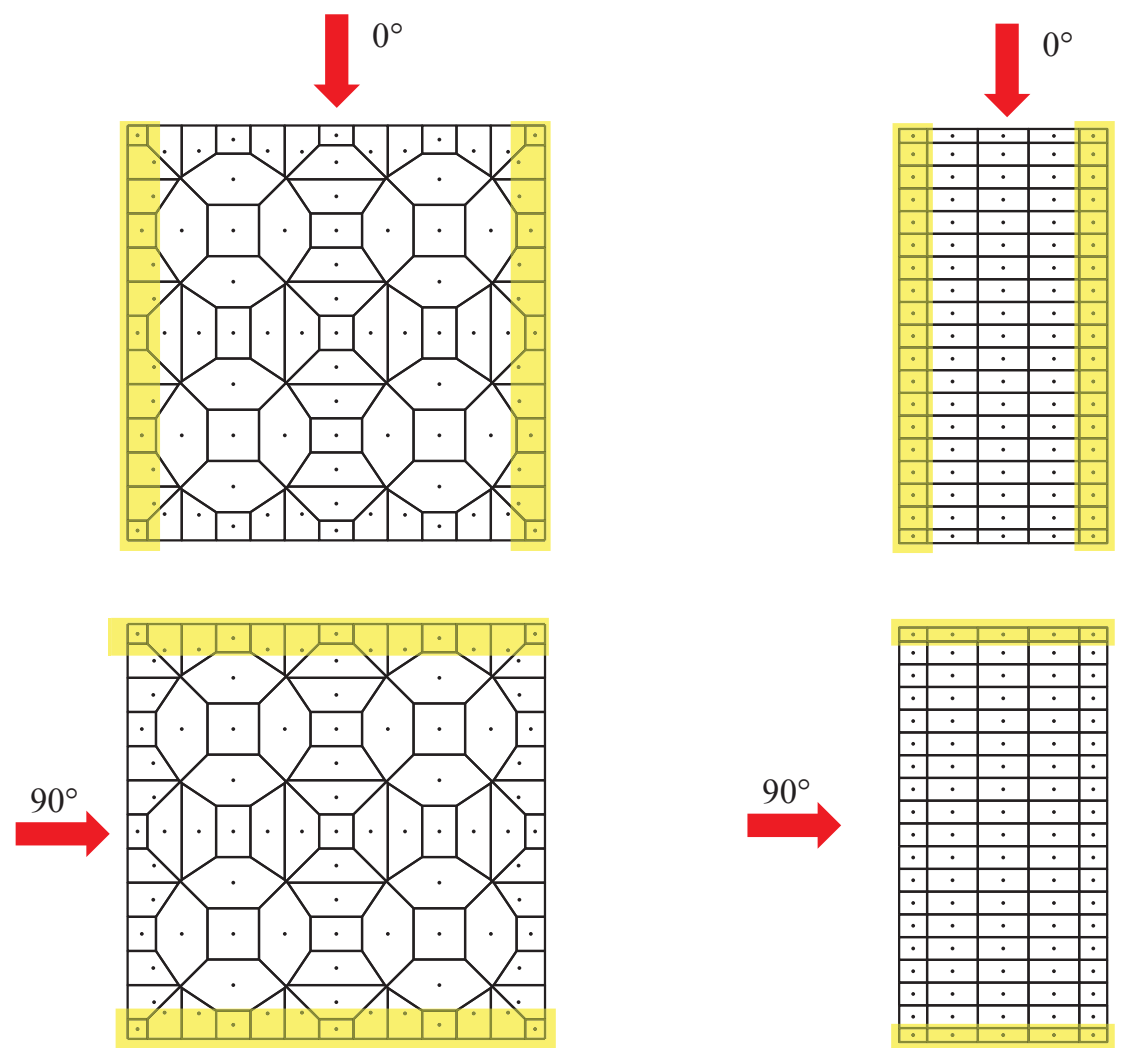

Figure 2: Map of the pressure taps for square and rectangular plans. The two alignments of pressure taps on the left and right edges of the roof with respect to the mean wind speed are highlighted in yellow 
For each wind direction along a symmetry axis (angle $0^{\circ}$ and $90^{\circ}$ ), Figure 2 reports the two alignments of pressure taps (highlighted in yellow) on the left and right edges of the roof with respect to wind direction, whose measured pressure coefficients will be compared to each other to discuss asymmetries shown by experimental tests.
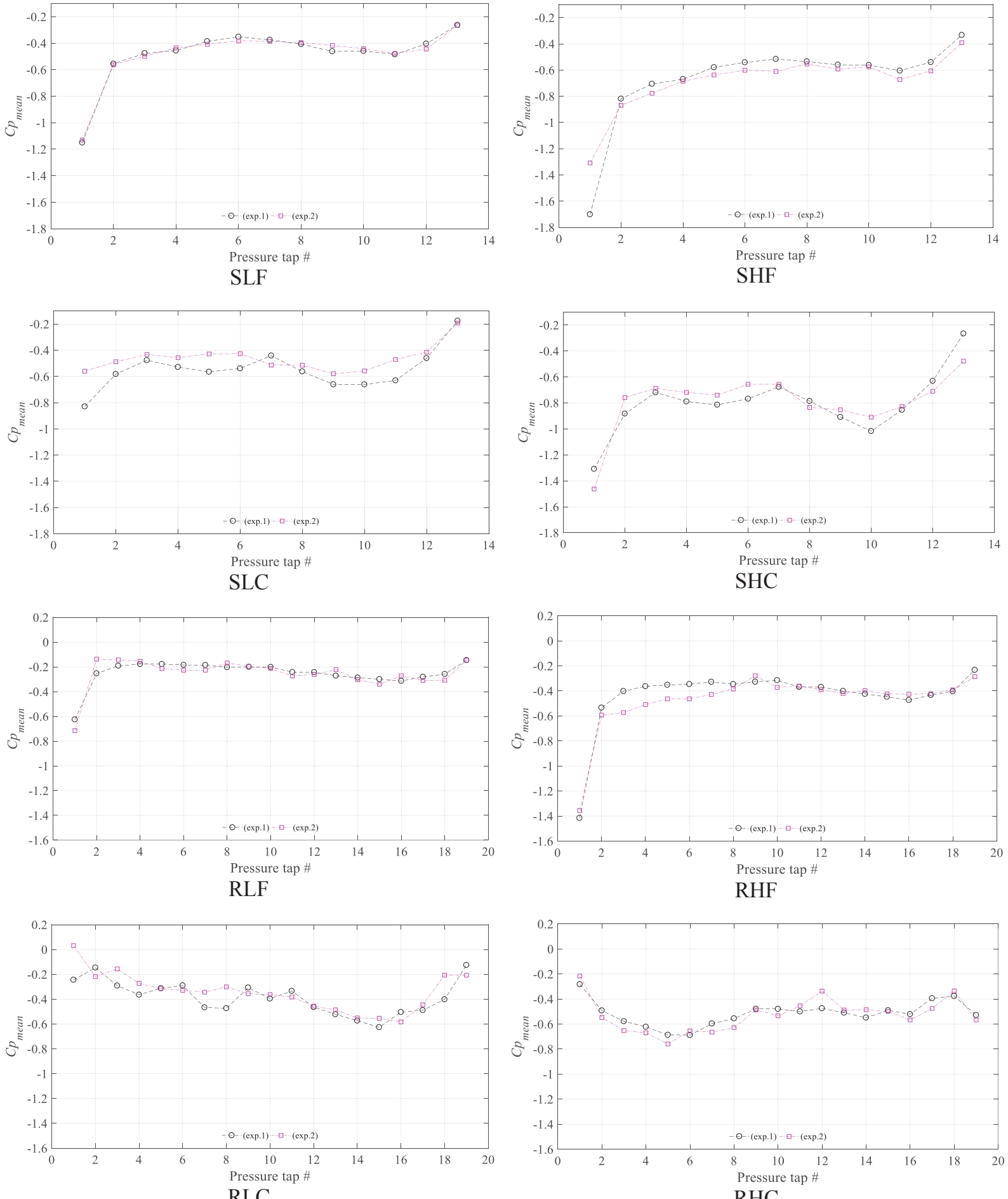

Figure 3: mean value $c_{p, \text { mean }}$ of the experimental $c_{p}$ for the eight models in fig. 1 and $\theta=0^{\circ}$, measured by the pressure taps along the roof sides on the left (black curve) and on the right (red curve) of the wind direction. 

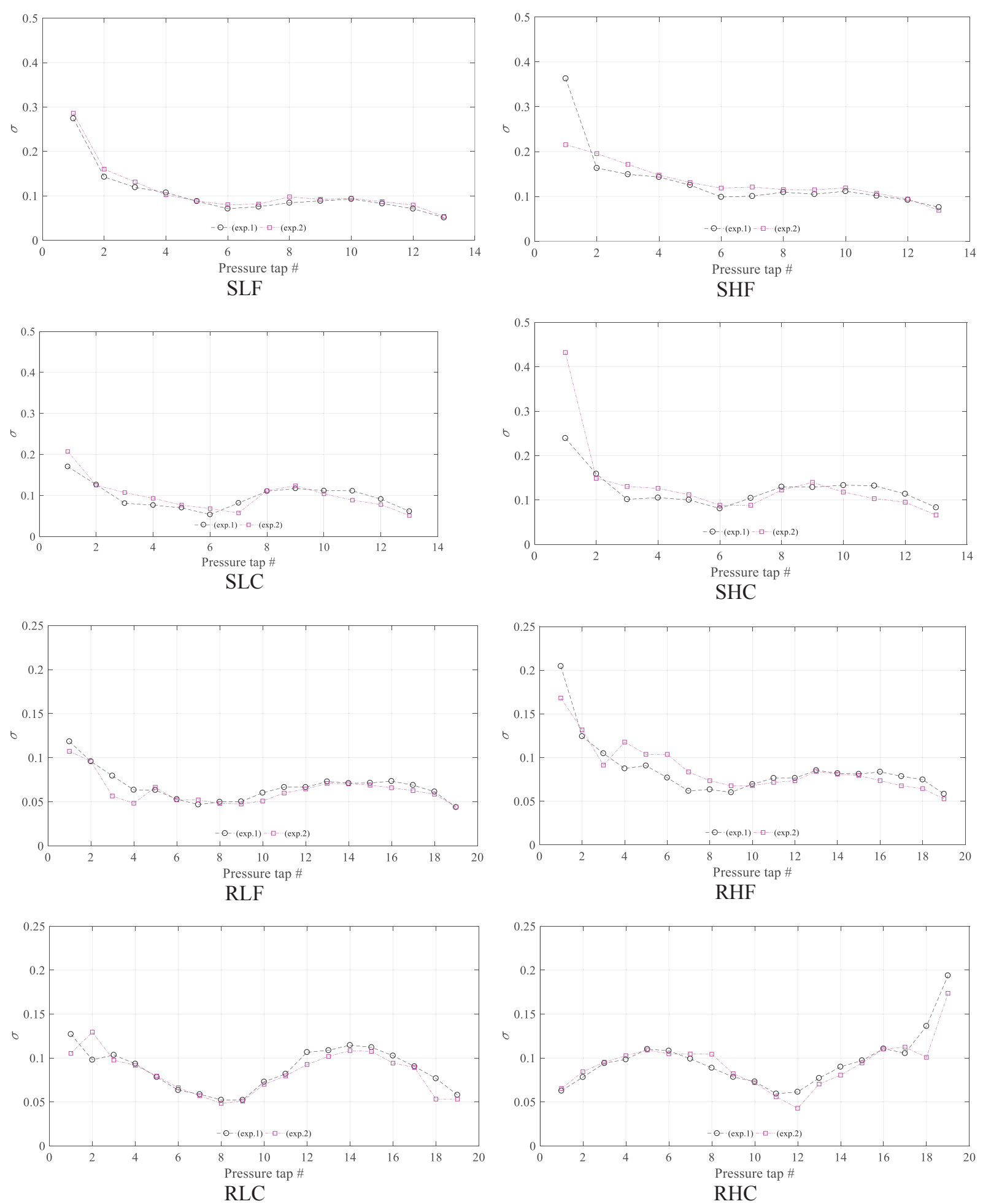

Figure 4: standard deviation $\sigma_{\mathrm{cp}}$ of the experimental $c_{\mathrm{p}}$ for the eight models in fig. 1 and $\theta=0^{\circ}$, measured by the pressure taps along the roof sides on the left (black curve) and on the right (red curve) of the wind direction.

\section{EXPERIMENTAL AND ANALYTICAL PEAK PRESSURE COEFFICIENTS}

Fig. 3 and 4 report the mean $c_{p \text {,mean }}$ and the standard deviation $\sigma_{\mathrm{cp}}$, respectively, of the experimental $c_{p}$ for the eight models in fig. 1 and $\theta=0^{\circ}$, measured by the pressure taps along the roof sides on the left and on the right of the wind direction. 
It can be observed that for $\theta=0^{\circ}$ the differences between the mean $c_{p, \text { mean }}$ of the experimental pressure coefficients (fig. 3) measured by the pressure taps along the two sides of the roof are almost negligible for low curvature roofs (SLF, SHF, RLF, RHF). This can easily be explained once considered that for $\theta=0^{\circ}$ the wind flow impacts on the hogging curvature of the model and it is confined by the across-wind sagging curvature, and this implies small detachment regions. As also expected, on the other hand, for more curved models (SLC, SHC, RLC, RHC) the differences become more relevant, because of more pronounced detachment regions of the flow due to the larger curvature.

A similar trend has been observed for the standard deviations $\sigma_{c p}$ of the experimental pressure coefficients (fig. 4).

The behavior becomes quite different for wind angle $\theta=90^{\circ}$, when the flow impacts the models on their highest wall, with a consequent detachment of the flow for a large part of the roof. In this case, as shown by results for square models reported in fig. 5 and 6 ( $\mathrm{c}_{\mathrm{p} \text {,mean }}$ and $\sigma_{\mathrm{cp}}$, respectively), the differences between the two alignments of pressure taps are still negligible for the lower model with smaller curvature (SLF). They are instead significantly larger for more curved ones, as shown by both $\mathrm{c}_{\mathrm{p} \text {,mean }}$ and $\sigma_{\mathrm{cp}}$ for SLC and SHC, and also for the highest model with smaller curvature (SHF), where significant differences of $\sigma_{\mathrm{cp}}$ have been found.
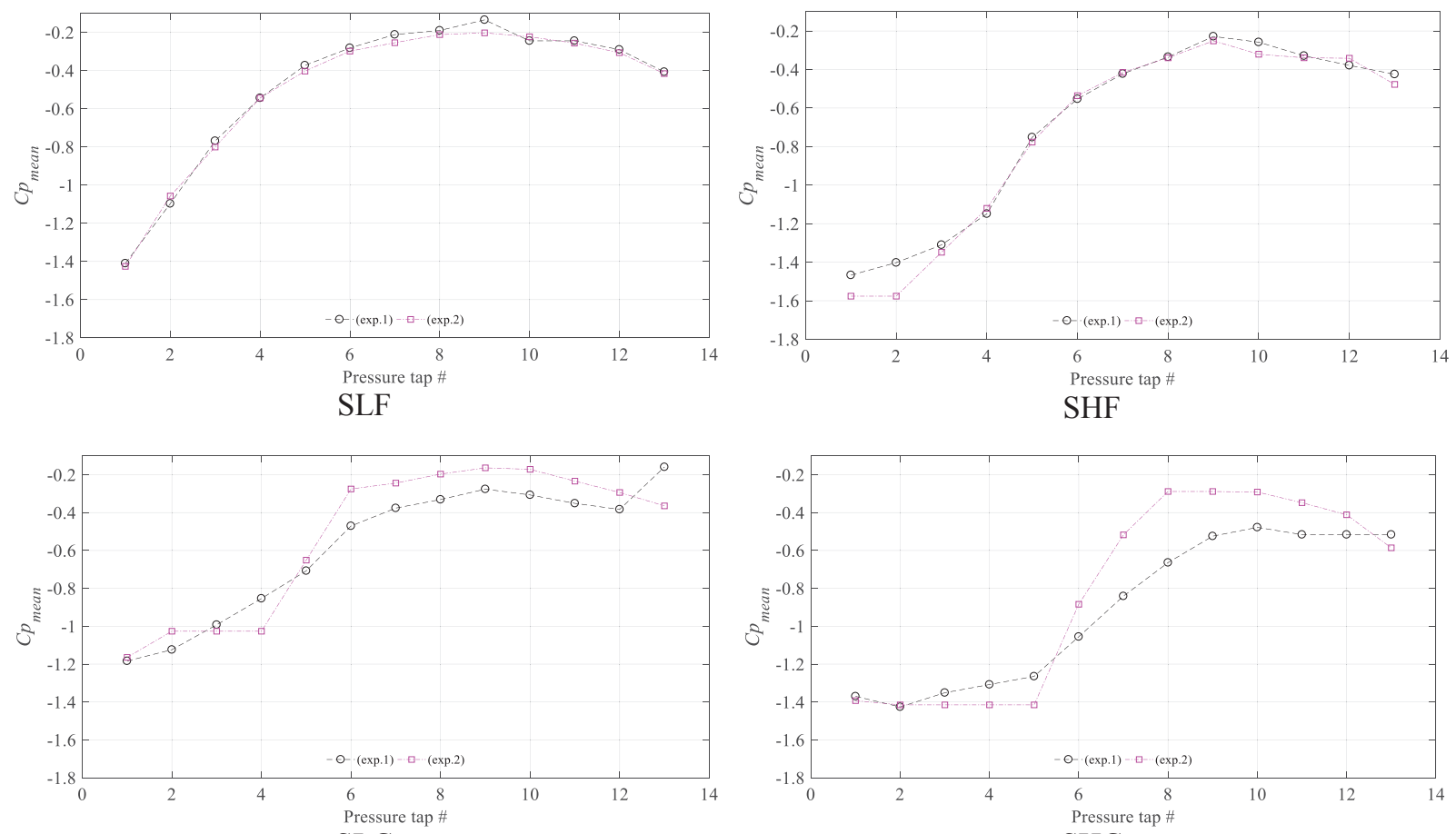

SLC

$\mathrm{SHC}$

Figure 5: mean value $c_{p \text {,mean }}$ of the experimental $c_{p}$ for the four square models in fig. 1 and $\theta=90^{\circ}$, measured by the pressure taps along the roof sides on the left (black curve) and on the right (red curve) of the wind direction.

Due to the experimentally observed non-symmetric behavior already discussed with reference to $c_{p \text {,mean }}$ and $\sigma_{\mathrm{cp}}$, the differences between minimum (suction) values of $\mathrm{c}_{\mathrm{p}}$ along the two alignments of pressure taps follow a similar trend or they are even more large, as shown for example by the extreme values in fig. 7 for square models and $\theta=0^{\circ}$.

The difference is even more evident for the experimental values of the peak factor $g$ corresponding to the minimum values of $\mathrm{c}_{\mathrm{p}}$ (i.e. suction), reported in fig. 8. 
Peak factor $g$ is defined as

$$
\mathrm{c}_{p}{ }^{\min }=\mathrm{c}_{p, \text { mean }}-g \sigma_{\mathrm{c} p}
$$

where for each pressure tap $c_{p \text {,mean }}, \sigma_{c p}$ and $\mathrm{c}_{p}$ min are the mean, the standard deviation and the minimum value of the $\mathrm{c}_{\mathrm{p}}$ time history recorded in wind-tunnel tests, respectively.

On the other hand, for the sample cases discussed here also maximum values of $c_{p}$ are almost always negative (suction) and smaller in absolute value than minima, and therefore they have no practical relevance to design purpose and are no further discussed below.

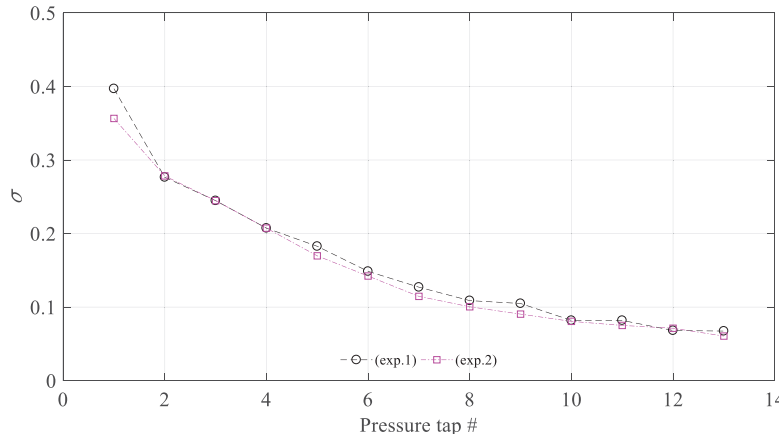

SLF

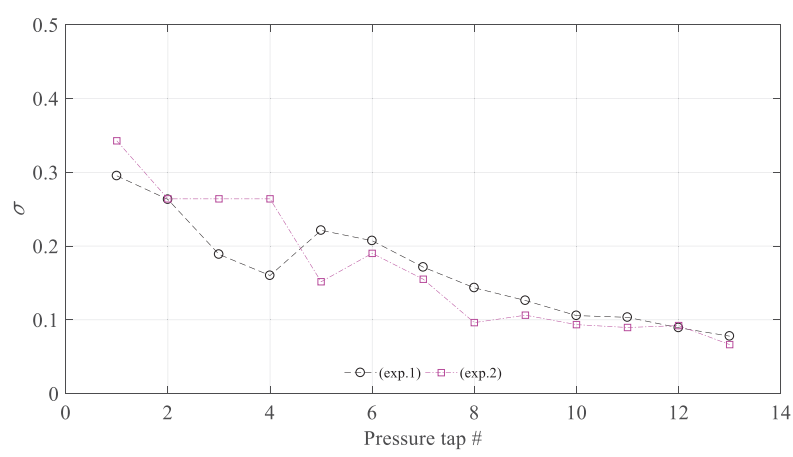

SLC

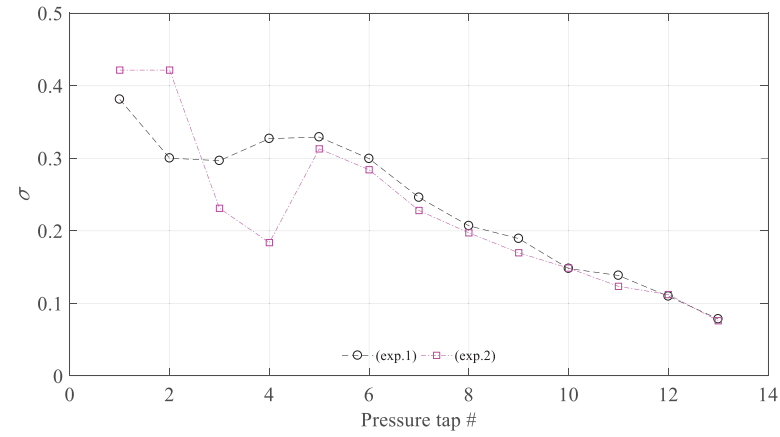

SHF

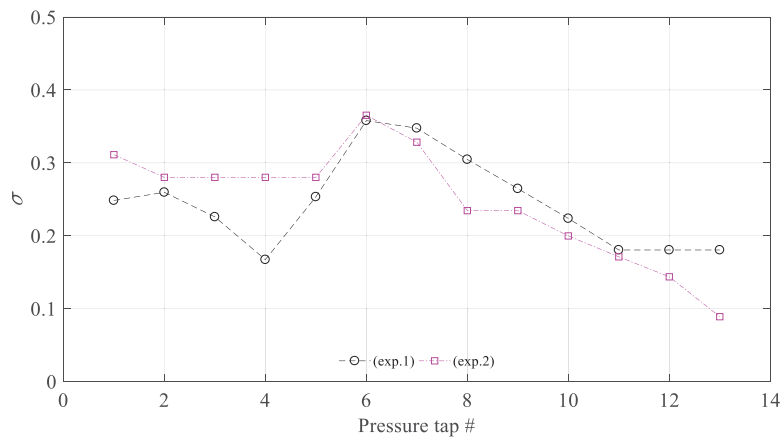

$\mathrm{SHC}$

Figure 6: standard deviation $\sigma_{\mathrm{cp}}$ of the experimental $\mathrm{c}_{\mathrm{p}}$ for the four square models in fig. 1 and $\theta=90^{\circ}$, measured by the pressure taps along the roof sides on the left (black curve) and on the right (red curve) of the wind direction.

Figures 7 also show for square models and $\theta=0^{\circ}$ the minimum values of $c_{p}$ evaluated according to the classical Davenport model and to the analytical modified Hermite model [1]. Similarly, fig. 8 also show for square models and $\theta=0^{\circ}$ the peak factor $g$ evaluated according to the classical Davenport model and to the modified Hermite model [1]. As expected, the Davenport peak factor assume almost constant values around 3.5.

It can be observed that, when compared to experimental results, the analytical models generally reduce the differences between the extreme values $\mathrm{c}_{p}{ }^{\mathrm{min}}$ of the two alignments of pressure taps. This is not true for the peak factor $g$ evaluated according to the analytical modified Hermite model [1], which keeps maintaining significant differences between taps expected to have a symmetric behavior. In the authors' opinion, however, this difference should be reduced by evaluating $\mathrm{g}$ from the minimum expected values of $\mathrm{c}_{\mathrm{p}}$ according to a soundly based statistical procedure (e.g. Cook and Mayne [12]), instead of assuming the minimum recorded values $\mathrm{C}_{p}{ }^{\mathrm{min}}$ as done here, and this will be investigated in future research.

Although differences between the two alignments are not negligible, however, the average value of $\mathrm{c}_{p}{ }^{\mathrm{min}}$ provided by the modified Hermite model [1] for pressure taps nominally symmetric is closer, with respect to the estimation provided by the Davenport model, to the aver- 
age of their recorded extreme suction values. This can be explained by considering that in the Davenport model $g$ turns out to be almost constant, while a recent research [4] shows that for this kind of geometries g may have quite large variations.

All in all, therefore, and according to these results for symmetrical geometries under wind flow direction parallel to the symmetry axis, asymmetries shown by experimental tests may be reduced by appropriate analytical models, that can therefore contribute to filter out the effects of these and others experimental uncertainties for design purposes.
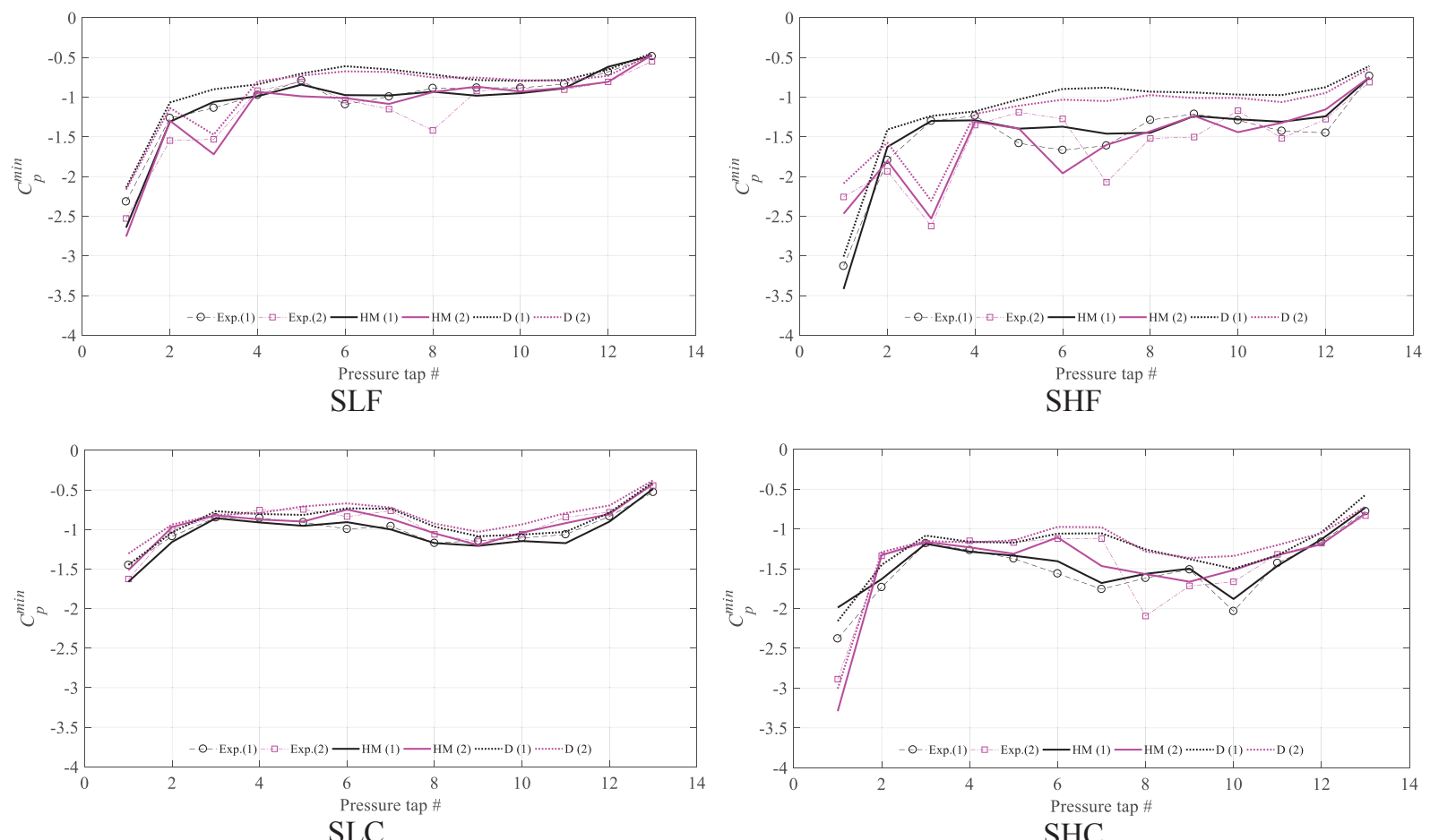

Figure 7: minimum value $c_{p}{ }^{\text {min }}$ of the experimental $c_{p}$ for the four square models in fig. 1 and $\theta=0^{\circ}$, measured by the pressure taps on the left (broken black curve) and on the right (broken red curve) of the wind direction; peak values of $c_{p}$ obtained with analytical models (Davenport and modified Hermite [1]) are also shown (dotted and continuous lines, respectively).

\section{CONCLUSIONS}

- Based on experimental results on hyperbolic paraboloid models tested in the boundary layer wind-tunnel of the CRIACIV (Italy) [4-9], this paper shows that experimental values of pressure coefficients are affected by physiological uncertainties, as the asymmetries measured for geometries and wind directions nominally symmetric.

- Although the results reported require further investigation, they suggest that technical codes should include asymmetrical load conditions also in case of nominally symmetric geometries and wind directions.

- Experimental peak pressure coefficients and their prediction given by two analytical models are compared for symmetrical geometries under wind flow direction parallel to the symmetry axis, and it is noted that asymmetries shown by experimental tests may be reduced by appropriate analytical models, that can therefore contribute to filter out the effects of experimental uncertainties for design purposes. 

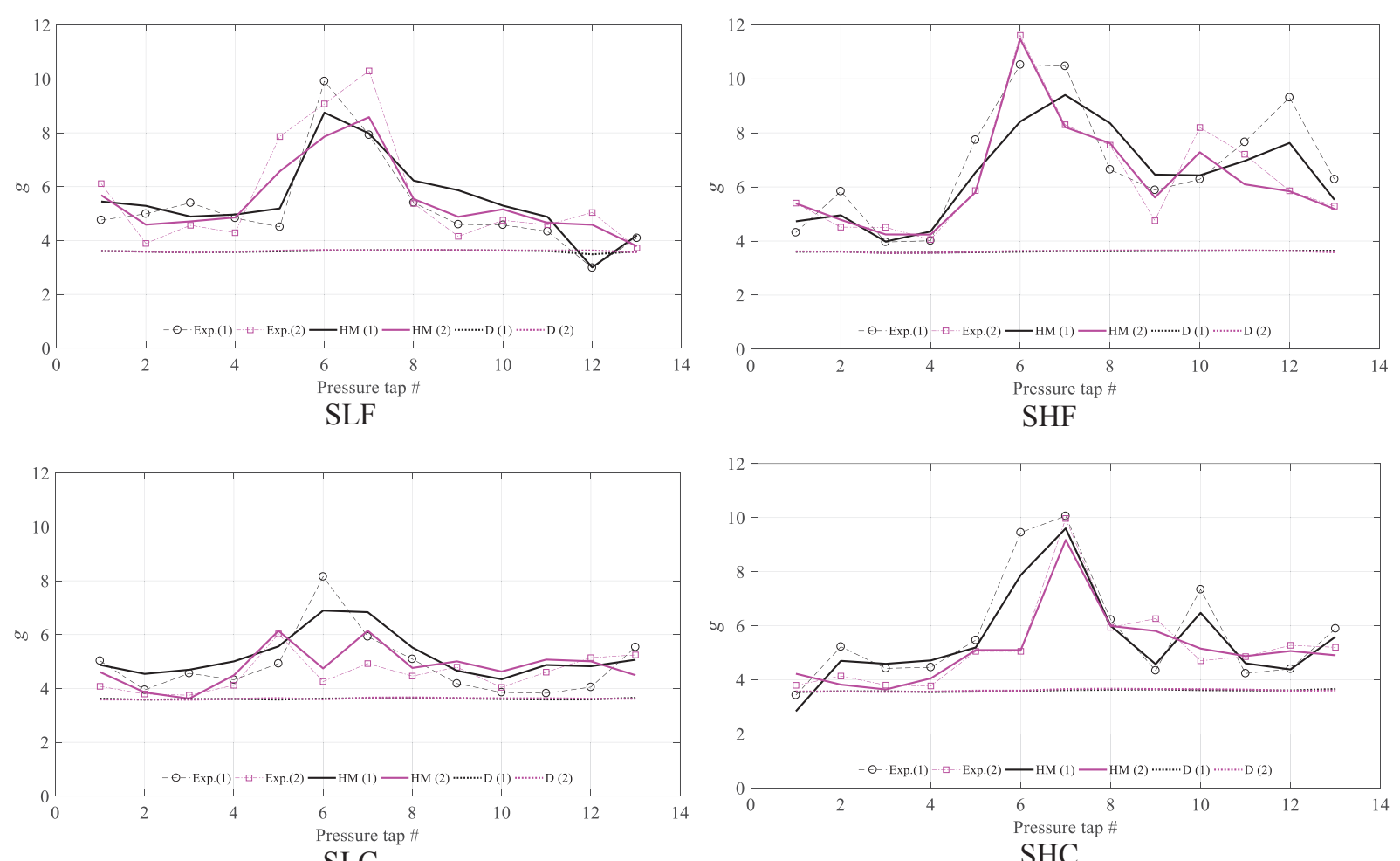

SLC

$\mathrm{SHC}$

Figure 8: peak factor $\mathrm{g}$ for the minimum values (suction) of the experimental $\mathrm{c}_{\mathrm{p}}$ for the four square models in fig. 1 and $\theta=0^{\circ}$, measured by the pressure taps along the roof sides on the left (broken black curve) and on the right (broken red curve) of the wind direction; peak factor $\mathrm{g}$ obtained with analytical models (Davenport and modified Hermite [1]) are also shown (dotted and continuous lines, respectively).

\section{ACKNOWLEDGMENTS}

The financial support of University "G. D'Annunzio" of Chieti-Pescara is acknowledged by the authors. Authors would like to thanks Professor Michele Barbato, UC-Davis University, California, USA, for sharing his expertise about the peak factors statistics and their prediction through analytical models.

\section{REFERENCES}

[1] D. Kwon, A. Kareem, Peak factors for non-Gaussian load effects revisited. J Struct Eng, 137(12):1611-1619, 2011.

[2] M. F. Huang, W. Lou, C. M. Chan, N. Lin, X. Pan, Peak distributions and peak factors of wind-induced pressure processes on tall buildings. J Eng Mech 139(12):1744-56, 2013.

[3] M. Liu, X. Chen, Q. Yang, Estimation of peak factor of non-Gaussian wind pressures by improved moment-based Hermite model, Journal of Engineering Mechanics, 143(7), 06017006-1-9, 2017.

[4] F. Rizzo, M. Barbato, V. Sepe, Peak factor statistics of wind effects for hyperbolic paraboloid roofs, Engineering Structures, 173, 313-330, 2018. 
[5] F. Rizzo, V. Sepe, M. Vasta, Correlation structure of wind-tunnel pressure fields for a hyperbolic paraboloid roof, XXIII Conference of the Italian Association of Theoretical and Applied Mechanics (AIMETA 2017), Salerno, Italy, September 4-7, 2017.

[6] F. Rizzo, P. D’Asdia, M. Lazzari, L. Procino, Wind action evaluation on tension roofs of hyperbolic paraboloid shape. Engineering Structures, 33 (2), 445-461, 2011.

[7] F. Rizzo, P. D’Asdia, F. Ricciardelli, G. Bartoli, Characterization of pressure coefficients on hyperbolic paraboloid roofs. Journal of Wind Engineering \& Industrial Aerodynamics, 102, 61-71, 2012.

[8] F. Rizzo, V. Sepe, Static loads to simulate dynamic effects of wind on hyperbolic paraboloid roofs with square plan. Journal of Wind Engineering \& Industrial Aerodynamics, 137-146, 2015.

[9] F. Rizzo, F. Ricciardelli, Design pressure coefficients for circular and elliptical plan structures with hyperbolic paraboloid roof. Engineering Structures, 139, 153-169, 2017.

[10] F. Rizzo, P. Zazzini, Improving the acoustical properties of an elliptical plan space with a cable net membrane roof, Journal of Acoustics Australia, 44, 449-456, (11-16-2016) ISSN: 08146039, DOI: 10.1007/s40857-016-0072-5, 2016

[11] F. Rizzo, P. Zazzini, Shape dependence of acoustic performances in buildings with a Hyperbolic Paraboloid cable net membrane roof. Journal of Acoustics Australia, 45 (2), (01-01-2017) 421-443, ISSN: 08146039, DOI: 10.1007/s40857-017-0092-9, 2017

[12] N.J. Cook, Mayne, J.R., A novel working approach to the assessment of wind loads for equivalent static design, J. Wind Eng. Ind. Aerod., 4(2), 149-164. https://doi.org/10.1016/0167-6105(79)90043-6, 1979. 